\title{
Electroacupuncture attenuates cognition impairment via anti-neuroinflammation in an Alzheimer's disease animal model
}

\author{
Mudan Cai', Jun-Hwan Lee ${ }^{2}$ and Eun Jin Yang ${ }^{2^{*}}$
}

\begin{abstract}
Background: Alzheimer's disease (AD) is a neurodegenerative disorder characterized by progressive loss of cognitive abilities and memory leading to dementia. Electroacupuncture (EA) is a complementary alternative medicine approach, applying an electrical current to acupuncture points. In clinical and animal studies, EA causes cognitive improvements in $A D$ and vascular dementia. However, EA-induced changes in cognition and microglia-mediated amyloid $\beta$ (A $\beta$ ) degradation have not been determined yet in $A D$ animals. Therefore, this study investigated the EA-induced molecular mechanisms causing cognitive improvement and anti-inflammatory activity in five familial mutation (5XFAD) mice, an animal model of AD.
\end{abstract}

Methods: 5XFAD mice were bilaterally treated with EA at the Taegye (KI3) acupoints three times per week for 2 weeks. To evaluate the effects of EA treatment on cognitive functions, novel object recognition and Y-maze tests were performed with non-Tg, 5XFAD (Tg), and EA-treated 5XFAD (Tg + KI3) mice. To examine the molecular mechanisms underlying EA effects, western blots, immunohistochemistry, and micro-positron emission tomography scans were performed. Furthermore, we studied synapse ultrastructures with transmission electron microscopy and used electrophysiology to investigate EA effects on synaptic plasticity in 5XFAD mice.

Results: EA treatment significantly improved working memory and synaptic plasticity, alleviated neuroinflammation, and reduced ultrastructural degradation of synapses via upregulation of synaptophysin and postsynaptic density-95 protein in 5XFAD mice. Furthermore, microglia-mediated A deposition was reduced after EA treatment and coincided with a reduction in amyloid precursor protein.

Conclusions: Our findings demonstrate that EA treatment ameliorates cognitive impairment via inhibition of synaptic degeneration and neuroinflammation in a mouse model of AD.

Keywords: Alzheimer's disease, Electroacupuncture, Cognitive function, Neuroinflammation

\section{Background}

Alzheimer's disease (AD) is a neurodegenerative disease characterized by progressive loss of cognitive and memory functions leading to dementia. The main pathological hallmarks of $\mathrm{AD}$ are extracellular amyloid plaques, intraneuronal accumulation of hyperphosphorylated tau proteins, and neuroinflammation $[1,2]$, but the precise pathophysiology in all its complexity remains elusive. Although several attempts have been made to develop drugs that target

\footnotetext{
* Correspondence: yej4823@gmail.com

${ }^{2}$ Department of Clinical Research, Korea Institute of Oriental Medicine, 1672

Yuseong-daero, Yuseong-gu, Daejeon 305-811, Republic of Korea

Full list of author information is available at the end of the article
}

amyloid plaques or hyperphosphorylated tau proteins, there is still no effective treatment option for AD patients.

Neuroinflammation occurs near amyloid plaques and hyperphosphorylated tau sequesters and leads to microand astrogliosis as its pathological markers [3]. Microglial cells are mainly involved in neuroinflammatory processes, and microglia dysfunction is related to $\mathrm{AD}$ development [4]. Microglial cells surround amyloid plaques and hyperphosphorylated tau aggregates, and microglial phagocytosis prevents $\mathrm{AD}$ progression $[5,6]$. Microglia also have critical roles in the regulation of synapse elimination, a critical process for precise synaptic connectivity and synaptic plasticity [7]. In addition, astrocytes activated by

(C) The Author(s). 2019 Open Access This article is distributed under the terms of the Creative Commons Attribution 4.0 International License (http://creativecommons.org/licenses/by/4.0/), which permits unrestricted use, distribution, and 
AD-associated neuroinflammation also influence synaptic communication [8] and synaptic apoptosis, thus contributing to the initial cognitive decline in $\mathrm{AD}$ [9]. Reactive astrocytes produce pro-inflammatory factors including tumor necrosis factor (TNF) $-\alpha$, interleukin (IL)-1 $\beta$, and cyclooxygenase-2 (COX2) causing synaptic disturbances and neuritic dystrophy in an AD animal model [10].

Electroacupuncture (EA) is one of the complementary alternative medicine techniques. It uses the acupuncture method by applying an electrical current to acupuncture needles. The effectiveness of EA for cognitive improvement in $\mathrm{AD}$ and vascular dementia has been proven in various clinical and animal studies [11, 12]. Some papers reported that the neuroprotective effects induced by EA are related to its anti-inflammatory activity [11, 12]. Feng et al. demonstrated that EA treatment suppresses the expression levels of pro-inflammatory cytokines including IL- $1 \alpha$, IL- 6 , and TNF- $\alpha$ that are related to the observed cognitive decline [13]. However, the direct influence of EA treatment on cognition and neuroinflammation in $\mathrm{AD}$ animals has not been elucidated yet. Therefore, the current study investigated the molecular mechanisms of EA effects causing cognitive improvement and anti-inflammatory actions in an animal model of $\mathrm{AD}$, the transgenic mice expressing five familial mutations (5XFAD) linked to $\mathrm{AD}$. We found that $\mathrm{EA}$ treatment attenuates cognitive impairment and neuroinflammation in 5XFAD mice. Furthermore, we demonstrate that EA treatment regulates microglia-mediated $\mathrm{A} \beta$ deposition in this animal model of AD. Taken together, we suggest that EA treatment can be useful to improve cognition in $\mathrm{AD}$ animals via anti-inflammatory effects.

\section{Methods}

\section{Animals}

5XFAD mice overexpress human amyloid precursor protein (APP) and presenilin-1 (PS1) mutants, namely the Swedish (K670 N, M671 L), Florida (I716V), and London (V717I) mutations in APP and the PS1 mutations M146 $\mathrm{L}$ and L286 V. Male 5XFAD mice and female B6/SJL were obtained from the Jackson Laboratory (Bar Harbor, ME, USA) and maintained in the Korea Institute of Oriental Medicine (KIOM). The offspring were genotyped by polymerase chain reaction analysis using tail DNA to identify mice with transgenic genes $[14,15]$. Four animals per cage were housed in an environment with a temperature of $21 \pm 3{ }^{\circ} \mathrm{C}$ and humidity of $50 \pm$ $10 \%$ with a 12-h light/dark cycle (light on 07:00-19:00 h). Access to water and food was ad libitum. Female transgenic mice were randomly assigned to two groups, either the group of 5XFAD transgenic mice $(\mathrm{Tg})$ or the group of 5XFAD transgenic mice treated with EA $(\mathrm{Tg}+$
KI3). Age-matched littermate B6/SJL mice of the nontransgenic group (non-Tg) were used as control.

All applicable international, national, and/or institutional guidelines for the care and use of animals were followed. Animals were kept in accordance with the animal care guidelines of the KIOM. All experiments were approved by the Institutional Animal Care and Use Committee (IACUC) of KIOM (approval number 18-033).

\section{Electroacupuncture (EA) treatment}

For EA stimulation, 6.5-month-old 5XFAD mice were subjected to EA treatment at the Taegye (KI3) acupoint. The KI3 acupoint was located on the medial aspect of the foot, between the medial malleolus and the Achilles tendon [16]. Acupuncture needles (diameter $0.18 \mathrm{~mm}$, length $8 \mathrm{~mm}$ ) were purchased from Dongbang Medical (Boryeong, Korea). All mice were anesthetized with $3 \%$ isoflurane (Hana Pharm Co. Ltd., Hwaseong, Korea) for induction and maintained during electrical stimulation with $1.5 \%$ isoflurane in a mixture of $70 \%$ nitrous oxide and 30\% oxygen. The anode and cathode of the electrical stimulator (Partner-1; Daejeon, South Korea) were connected to the two acupuncture needles. Acupuncture needles were inserted at a depth of $2 \mathrm{~mm}$, and electrical stimulation pulses were applied for $15 \mathrm{~min}(1 \mathrm{~mA}, 2 \mathrm{~Hz})$ at the bilateral KI3 acupoints. EA treatment was performed three times per week for 2 weeks.

\section{Novel object recognition (NOR) test}

The novel object recognition test, including habituation, training, and probe trial, was conducted for 3 days according to previously described methods $[17,18]$. On the first day, all mice were exposed individually to the test box $(40 \times 40 \times 40 \mathrm{~cm})$ for $10 \mathrm{~min}$ to habituate to the test environment without any objects. On the second day, the animals were placed again in the box to explore two identical familiar objects for $5 \mathrm{~min}$. In the probe test $24 \mathrm{~h}$ after the training trial, the animals were placed in the same box, one object was exchanged with a novel object of a different shape but a similar texture, and the mice were given $5 \mathrm{~min}$ to examine the items. Boxes were thoroughly cleaned with $70 \%$ ethanol to remove residual odors between test runs. The exploration time was recorded using a video camera, and the percentage of exploration time of the novel object was determined according to the following equation: $\left(T_{\text {novel }}\right) /\left(T_{\text {novel }}+\right.$ $\left.T_{\text {familiar }}\right) \times 100$. In this behavioral test, the total exploration time $\left(T_{\text {novel }}+T_{\text {familiar }}\right)$ was expected to be $>10 \mathrm{~s}$ in the probe trial; if the total exploration time was less than $10 \mathrm{~s}$, this mouse was not included in the analysis.

\section{Y-maze test}

The Y-maze test was performed the day after the last EA treatment as described previously [19]. The Y-maze was a Y-shaped maze made of black polyvinyl chloride 
plastic $(40 \mathrm{~cm}$ long and $3 \mathrm{~cm}$ wide with $15-\mathrm{cm}$-high walls) in which the arms are at $120^{\circ}$ angles from each other. Mice were put into the center of the maze and allowed to explore it. The numbers of entries and the sequence of entered arms were manually recorded for 8 min. A spontaneous alternation was defined as entries into three different arms consecutively (i.e., $A B C, C A B$, or $\mathrm{BCA}$, but not $\mathrm{BAB}$ and $\mathrm{CBC}$ ). The $\mathrm{Y}$-maze arms were thoroughly cleaned with $70 \%$ ethanol to remove residual odors after each test. The calculation was defined as follows: percentage alternation $=$ (number of alternations) $/$ (total arm entries -2 ) $\times 100$. The number of total arm entries served as an indicator of locomotor activity.

\section{Western blots}

Western blotting was performed as described previously [20]. Prefrontal cortexes were lysed in radioimmunoprecipitation assay buffer (Biosesang, Gyeonggi-do, Korea) containing phosphatase and proteinase inhibitors (Thermo Fisher Scientific, Waltham, MA, USA). The total loading protein $(20 \mu \mathrm{g})$ was quantified using the Bicinchoninic Acid Assay Kit (Pierce, Rockford, IL, USA) and separated on Bolt 4-12\% Bis-Tris Plus gels (Thermo Fisher Scientific). The gels were transferred to polyvinylidene difluoride membranes (Bio-Rad, Hercules, CA, USA). Transferred membranes were incubated with primary antibodies overnight at $4{ }^{\circ} \mathrm{C}$. The next day, these membranes were incubated with secondary antibodies (Santa Cruz Biotechnology, Santa Cruz, CA, USA). The immunoblots were visualized using a ChemiDoc Imaging System device (Bio-Rad) and quantified using ImageJ (version 1.46j; National Institutes of Health, Bethesda, MD, USA). The primary antibodies used were as follows: cluster of differentiation 11b (CD11b), heme oxygenase (HO)-1, TNF- $\alpha$, COX2, and tubulin (all 1:1000; Abcam, Cambridge, MA, USA); B cell lymphoma-2-associated X protein (Bax), transferrin (all 1:1000; Santa Cruz Biotechnology); $\beta$-site amyloid precursor protein cleaving enzyme 1 (BACE), postsynaptic density protein 95 (PSD95; all 1:1000; Cell Signaling Technology, Danvers, MA, USA); APP, apolipoprotein E (APOE; all 1:1000; Merck Millipore, Burlington, MA, USA); and glial fibrillary acidic protein (GFAP, 1:5000; Agilent Technologies, Santa Clara, CA, USA).

\section{Electrophysiology}

Electrophysiology was performed at Dong-A University (Busan, Korea). Mice were anesthetized, and their brains were removed and transferred into cold artificial cerebrospinal fluid $\left(\mathrm{NaCl}, 124 \mathrm{mM} ; \mathrm{KCl}, 3 \mathrm{mM} ; \mathrm{NaHCO}_{3}\right.$, $26 \mathrm{mM} ; \mathrm{NaH}_{2} \mathrm{PO}_{4}, 1.25 \mathrm{mM} ; \mathrm{CaCl}_{2}, 2 \mathrm{mM} ; \mathrm{MgSO}_{4}, 1$ $\mathrm{mM}$; D-glucose, $10 \mathrm{mM}$; bubbled with $95 \% \mathrm{O}_{2} / 5 \% \mathrm{CO}_{2}$ ) at $24 \mathrm{~h}$ after the last EA treatment. The prefrontal cortex regions were isolated and cut into $400-\mu \mathrm{m}$ thick slices using a vibratome. Field excitatory postsynaptic potentials (fEPSPs) were recorded in layer $\mathrm{V}$ of the prefrontal cortex. After observing a stable baseline for $20 \mathrm{~min}$, long-term potentiation (LTP) was induced by highfrequency stimulation $(100$ pulses at $100 \mathrm{~Hz}, 5$ trains, 10-s intervals between stimulations of the prefrontal cortex), and fEPSPs were recorded at 2-min intervals for $100 \mathrm{~min}$. For quantification, the LTP ratios were averaged for the last $10 \mathrm{~min}$ of the fEPSP recordings.

\section{$A \beta_{1-42}$ enzyme-linked immunosorbent (ELISA) assays}

To determine the amounts of insoluble $A \beta_{1-42}$ in the prefrontal cortexes from mice of each group, a betaamyloid 1-42 quantitative ELISA kit was used (Anaspec Inc., Fremont, CA, USA). The prefrontal cortex tissue was homogenized with guanidine- $\mathrm{HCl}$ for $4 \mathrm{~h}$ at room temperature, thoroughly mixed, and centrifuged at 12 , $000 \mathrm{rpm}$ for $15 \mathrm{~min}$. The collected supernatants of the prefrontal cortexes were diluted 1:10000 in sample dilution buffer and measured according to the manufacturer's protocol.

\section{Immunohistochemistry and immunofluorescence staining}

For immunohistochemistry and immunofluorescence studies, mice were perfused with phosphate-buffered saline (PBS), and their brains were removed. The brains were fixed in $4 \%$ paraformaldehyde for 3 days at $4{ }^{\circ} \mathrm{C}$. Fixed brains were embedded in paraffin and sectioned to $10-\mu \mathrm{m}$ thickness. Immunohistochemistry and immunofluorescence studies were conducted as previously described [21].

For $A \beta$ immunohistochemistry staining, the slices were deparaffinized in xylene, rehydrated in graded alcohol solutions, and washed again in PBS. Sections were incubated in $90 \%$ formic acid for $5 \mathrm{~min}$ to retrieve the antigens before incubating them with primary antibodies against anti-A $\beta$ 4G8 (1:400; BioLegend, San Diego, CA, USA) overnight at $4{ }^{\circ} \mathrm{C}$. The next day, the sections were incubated with the matched secondary antibody before visualization of the antibody staining with Avidin-Biotin Complex solution (Vector Laboratories, Burlingame, CA, USA) followed by 3,3'-diaminobenzidine peroxidase substrate solution (Vector Laboratories). For quantification of $A \beta$ plaques, images of the slices were captured using a microscope (Olympus BX53, Tokyo, Japan), and the cellSens software was used to analyze the diameter of $A \beta$ plaques in the captured image. In this study, immunoreactive $A \beta$ was counted if an $A \beta$ plaque had a diameter $>40 \mu \mathrm{m}$.

For double immunofluorescence staining, brain slices were incubated in formic acid for antigen retrieval and blocked in $5 \%$ bovine serum albumin. The tissue samples were incubated overnight at $4{ }^{\circ} \mathrm{C}$ with the following primary antibodies: rabbit anti-CD68 antibody (1:500; 
Abcam) and mouse anti-A $\beta$ (4G8) antibody (1:400; BioLegend). On the next day, the sections were incubated with a secondary antibody conjugated to goat-antimouse Alexa 488 or goat-anti-rabbit Alexa 598 (1:1000; Invitrogen, Carlsbad, CA, USA), then washed and mounted on glass slides using Vectashield mounting medium (Vector Laboratories). For quantification, images were taken with a fluorescence microscope (Olympus BX53) and the numbers of A $\beta$-positive plaques and CD68-positive microglial cells were counted.

\section{MicroPET scanning}

MicroPET imaging was performed at the Osong Medical Innovation Foundation (Osong-eup, Chung Buk, South Korea). An animal positron-emission tomography (PET) scanner (Nano PET/CT; Mediso, Hungary) was used for imaging of mice under inhalation anesthesia (1.5-2.0\% of isoflurane in $1.0-1.5 \mathrm{l} / \mathrm{min}$ of oxygen). For microPET scanning, mice were food-restricted for over $8 \mathrm{~h}$ and anesthetized, and fluorodeoxyglucose $\left({ }^{18} \mathrm{~F}\right.$-FDG; $7.4 \mathrm{MBq}$ in $0.1 \mathrm{ml}$ of saline solution) was intravenously injected via the tail vein. Then, 60 min after the injection of ${ }^{18}$ F-FDG, PET images were acquired for $30 \mathrm{~min}$ using the following parameters: axial field of view $94.7 \mathrm{~mm}$ (1 bed), 1-5 acquisition coincidence mode, and coincidence time window of $5 \mathrm{~ns}$. The sinogram of the images was reconstructed using the $3 \mathrm{D}$ expectation maximization PET reconstruction algorithm (Mediso TeraTomo TM, iteration $=4$, subset $=3$ ). All PET images were corrected for emission scatter and attenuation. To the quantitative analysis, regions of interest (ROIs) were drawn into the frontal cortex, cortex, hippocampus, and hypothalamus regions using PMOD 3.8 software (PMOD Technologies LLC., Zurich, Switzerland). Subsequently, the regional uptake was calculated as the mean standardized uptake value (SUV) based on the following equation for the quantitative comparison of ${ }^{18} \mathrm{~F}$-FDG uptake.

$$
S U V=\frac{\text { tissue radioactivity concentration }}{\text { injected activity } / \text { body weight }}
$$

\section{Transmission electron microscopy (TEM)}

Prefrontal cortex samples were cut into $1-\mathrm{mm}^{3}$ cubes and immediately fixed in 2.5\% glutaraldehyde (Sigma-Aldrich, St. Louis, MO, USA) in $0.1 \mathrm{M}$ phosphate buffer (pH 7.2). Then, samples were postfixed in $4 \%$ osmium tetroxide (Sigma-Aldrich) with $3 \%$ potassium ferrocyanide in $0.1 \mathrm{M}$ cacodylate buffer ( $\mathrm{pH} 7.3$ ) for $1 \mathrm{~h}$ at $4{ }^{\circ} \mathrm{C}$ in the dark and embedded in Epon 812 after dehydration in an ethanol and propylene oxide series. Ultrathin slices (70 $\mathrm{nm}$ thick) were obtained using an ultramicrotome (Ultracut UCT, Leica, USA) and collected on 150 mesh copper grids. After staining with $2 \%$ uranyl acetate for $10 \mathrm{~min}$ and lead citrate for $5 \mathrm{~min}$, the sections were observed under a transmission electron microscopy (Tecnai G2 Spiri TWIN; Thermo Fisher Scientific) at $120 \mathrm{kV}$.

\section{Statistical analysis}

Data are presented as the mean \pm standard error of the mean (SEM). The results were analyzed by one-way analysis of variance followed by the Newman-Keuls post hoc test for multiple comparisons using Prism v.5.0 (GraphPad, La Jolla, CA, USA). Statistical significance thresholds were set at ${ }^{*} p<0.05,{ }^{* * *} p<0.01$, and ${ }^{* * *} p<$ 0.001 .

\section{Results}

EA attenuates cognitive impairments in 5XFAD mice

EA treatment at KI3 was performed in 6.5-month-old 5XFAD mice three times per week for 2 weeks (Fig. 1). NOR and Y-maze tests were used to evaluate whether EA treatment affects cognitive impairments in 5XFAD mice. We found that the Tg group of untreated 5XFAD mice showed a 1.4-fold reduction in exploration time of novel objects compared to that of non-Tg mice. However, EA treatment increased the mean exploration time of novel objects by 1.3 times compared to that of $\mathrm{Tg}$ mice in the NOR test that measures the working memory (Fig. 2a). In the Y-maze test, the total activity was not different among the three groups, non- $\mathrm{Tg}, \mathrm{Tg}$, and $\mathrm{Tg}+\mathrm{KI} 3$. In this test, 5XFAD mice showed a 1.3 -fold decrease in the number of spontaneous alternations compared to that of age-matched non- $\mathrm{Tg}$ mice indicating an impairment of the hippocampus-dependent spatial memory (Fig. 2b). However, EA treatment did not rescue the observed dysfunction of hippocampusdependent spatial memory in 5XFAD mice (Fig. 2b). Taken together, these data suggest that EA treatment restored in the 5XFAD model the working memory but not the spatial memory that is dependent on hippocampus functions.

\section{EA activates specific brain regions in 5XFAD mice}

Some papers reported that acupuncture at KI3 activates the brain to increase cognition in elderly patients with mild cognitive impairment (MCI) [22, 23]. To evaluate whether EA treatment activates the prefrontal cortex region involved in cognitive functions, we investigated glucose metabolism levels using microPET to measure brain functions in 5XFAD mice. As expected, EA stimulation caused a 1.1-fold increase in the mean glucose level of the frontal cortex, which is related to short-term and working memory, compared to that of the Tg group, whereas the levels in the hippocampus did not change (Fig. 3a, b). Furthermore, we observed that the hypothalamus, which is related to energy metabolism, exhibited a 1.1-fold decreased glucose metabolism in $\mathrm{Tg}$ mice 
- NOR probe test /Y-maze test

- MicroPET

- Electrophysiology

Sacrifice for IHC/WB/TEM

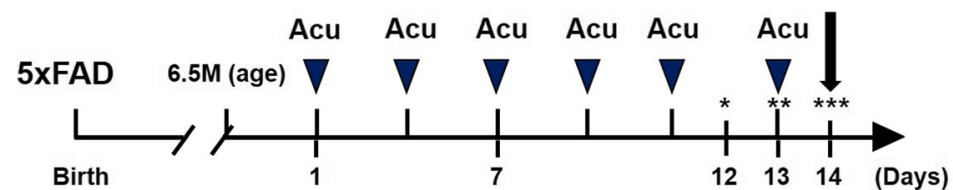

Fig. 1 Experimental procedures. NOR novel object recognition test, Acu acupuncture treatment, IHC immunohistochemistry, WB western blot, TEM transmission electron microscopy. An asterisk indicates habituation trial, two asterisks indicate training trial, and three asterisks show probe trial in the NOR test

compared to non-Tg mice, and this effect was fully reversed in EA-treated Tg mice (Fig. 3a, b). These data indicate that EA treatment leads to an activation of the brain metabolism in Tg mice.

\section{EA attenuates neuroinflammation in 5XFAD mice}

Neuroinflammation is a critical factor causing cognitive impairments in 5XFAD mice or AD [24]. To investigate the molecular mechanisms responsible for EA-induced the improvement of cognition, we examined the expression levels of neuroinflammation and oxidative stressrelated proteins in the prefrontal cortex of 5XFAD mice. As shown in Fig. 4a, b, the expression levels of CD11b (for microglia), GFAP (for astrocytes), and COX2 showed in the prefrontal cortex of EA-treated mice a significant 1.9-fold, 1.5-fold, and 1.6-fold reduction, respectively, in comparison to the values determined in $\mathrm{Tg}$ mice (Additional file 1: Figure S1b and S1d). In addition, EA treatment decreased the levels of the oxidationrelated proteins HO-1, transferrin, and Bax 2.1-fold, 1.5fold, and 1.8-fold, respectively, in the prefrontal cortexes of 5XFAD mice in comparison to those of Tg mice (Fig. $4 \mathrm{c}, \mathrm{d}$ and Additional file 1: Figure S1a-b). These data suggest that the observed cognitive improvement by EA treatment may be closely related to anti-inflammatory mechanisms in the brain.

\section{EA increases synaptic plasticity and reduces synaptic ultrastructural degradation in 5XFAD mice}

Neuroinflammation such as microglia activation leads to a decrease in synaptic plasticity and synapse loss [25]. To investigate the relationship between neuroinflammation and synaptic plasticity after EA treatment, we measured LTP and determined whether synaptic plasticity is influenced by the upregulation of synapse-related proteins in the murine prefrontal cortex. Prefrontal cortex and hippocampus slices of non-Tg mice presented the expected LTP levels (Fig. 5a). The mean LTP level in prefrontal cortex and hippocampus slices from 5XFAD mice showed a significant 1.2-fold and 1.4-fold reduction, respectively, compared to those from non-Tg mice. EA treatment enhanced the LTP level in the prefrontal cortex of 5XFAD mice without reaching statistical significance in comparison to untreated animals, but not in the hippocampus (Fig. 5a). In agreement with these data, we found that stimulation by EA led to a significant 1.4fold increase in the expression level of synaptophysin in the prefrontal cortex compared to that of untreated $\mathrm{Tg}$ mice (Fig. 5b and Additional file 1: Figure S1e). In addition, a significant 3.2-fold increase in PSD-95 protein level was found in EA-treated Tg mice compared to that of untreated $\mathrm{Tg}$ mice (Fig. $5 \mathrm{~b}$ and Additional file 1: Figure S1d). Next, we determined whether EA treatment
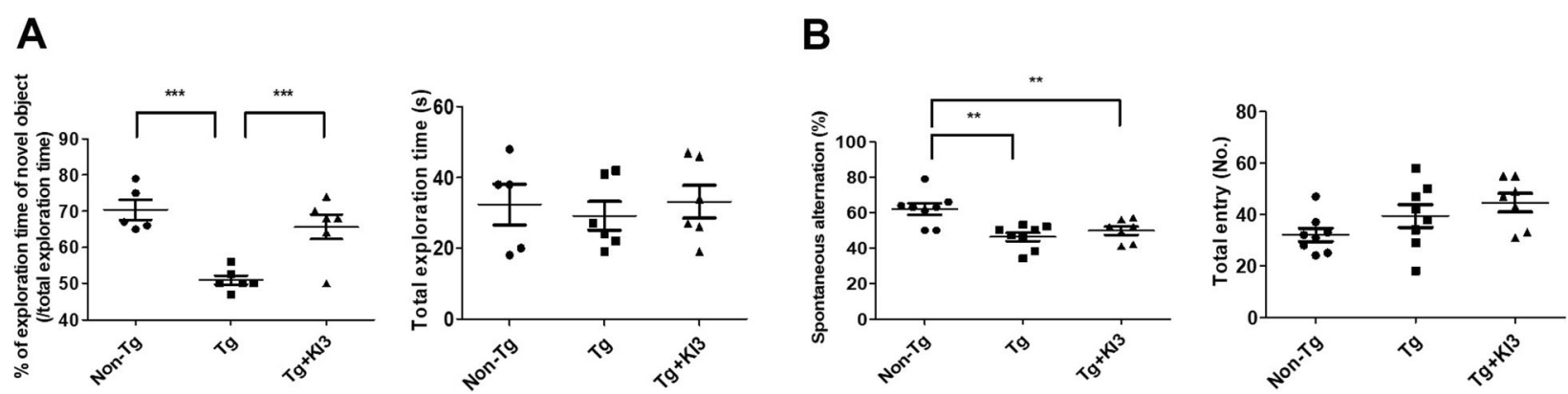

Fig. 2 EA treatment alleviates cognitive impairments in 5XFAD mice. EA was applied bilaterally to the KI3 acupoints for $15 \mathrm{~min}(1 \mathrm{~mA}, 2 \mathrm{~Hz})$ three times per week for 2 weeks. The NOR probe trial was conducted on the day after the last EA treatment. a Data show the results of the NOR test (percentage of exploration time of the novel object and total exploration time; $n=5-6 /$ group). b Results of the Y-maze test (spontaneous alternations and total entries; $n=7-8 /$ group $)$. Data are presented as means \pm SEM $\left({ }^{* *} p<0.01,{ }^{* * *} p<0.001\right)$ 


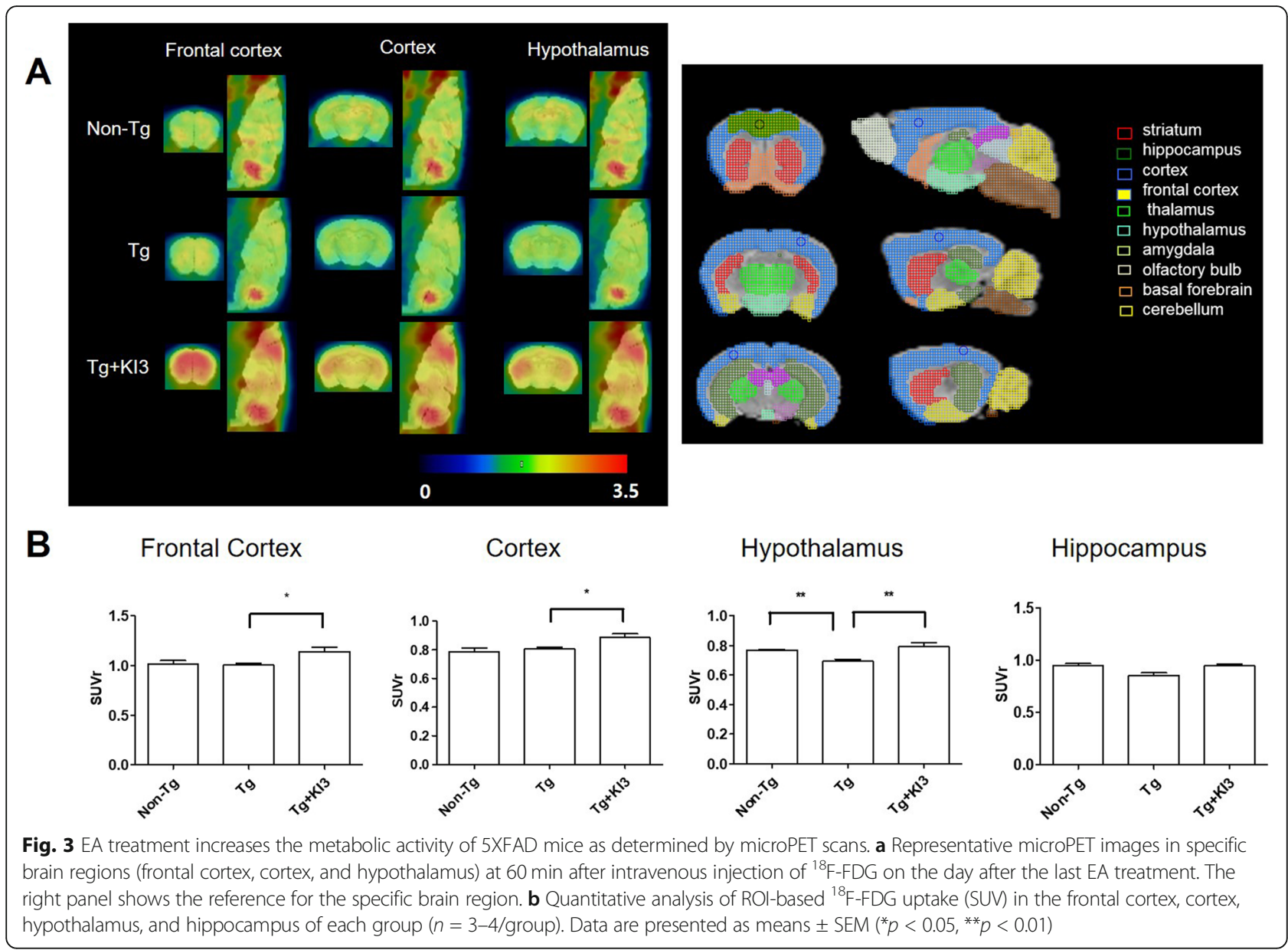

affects the synapse ultrastructure using transmission electron microscopy in the prefrontal cortex of 5XFAD mice. Synapses of non-Tg mice showed clear synaptic membranes with synaptic vesicles and postsynaptic densities (Fig. 5c). However, 5XFAD mice showed disrupted membranes at pre- and postsynaptic densities, fusion of the synaptic cleft, and loss of synaptic vesicles. By contrast, EA treatment clearly restored the visible synaptic structures compared to those observed in $\mathrm{Tg}$ mice (Fig. 5c). These findings indicate that EA upregulated the expression of synaptic proteins and inhibited the degradation of synaptic ultrastructures, thus improving synaptic plasticity.

\section{$E A$ modulates microglia-mediated $A \beta$ deposition in 5XFAD mice}

To determine whether EA affects $A \beta$ depositions in the prefrontal cortex of 5XFAD mice, we studied the protein expression levels related to $A \beta$ production and the amounts of insoluble $A \beta$ in western blots and ELISAs. As shown in Fig. 6a, the expression levels of proteins related to $A \beta_{1-42}$ production such as APP, APOE, and BACE showed a significant 4.3-, 5.4-, and 1.5-fold increase, respectively, in the prefrontal cortex of Tg mice relative to the endogenous levels of non-Tg mice (Additional file 1: Figure S1a and S1c). However, EA treatment caused a 1.5-fold reduction in the level of APP protein compared to that of untreated $\mathrm{Tg}$ mice. In addition, the levels of APOE and BACE were reduced after EA treatment without reaching statistical significance (Fig. 6a, b and Additional file 1: Figure S1c). To determine whether EA treatment affects insoluble $A \beta_{1-}$ ${ }_{42}$ levels and $A \beta_{1-42}$ plaques in the prefrontal cortex, we performed $A \beta_{1-42}$ ELISAs and immunohistochemistry. EA treatment significantly lowered 1.2-fold the insoluble $\mathrm{A} \beta_{1-42}$ levels in the prefrontal cortex of Tg mice (Fig. $6 c)$. In agreement with these ELISA results, EA treatment led in the prefrontal cortex to a 1.5-fold reduction in the number of $A \beta_{1-42}$ plaques with a diameter > $40 \mu \mathrm{m}$ compared to the values determined in untreated Tg mice (Fig. 6d). However, EA treatment did not reduce the number of $A \beta_{1-42}$ plaques in the hippocampus of Tg mice (Fig. 6d). Amyloid depositions are reportedly surrounded by activated microglia [26]. Based on this paper, we investigated the colocalization of $A \beta_{1-42}$ plaques and CD68-positive cells in the prefrontal cortex of 

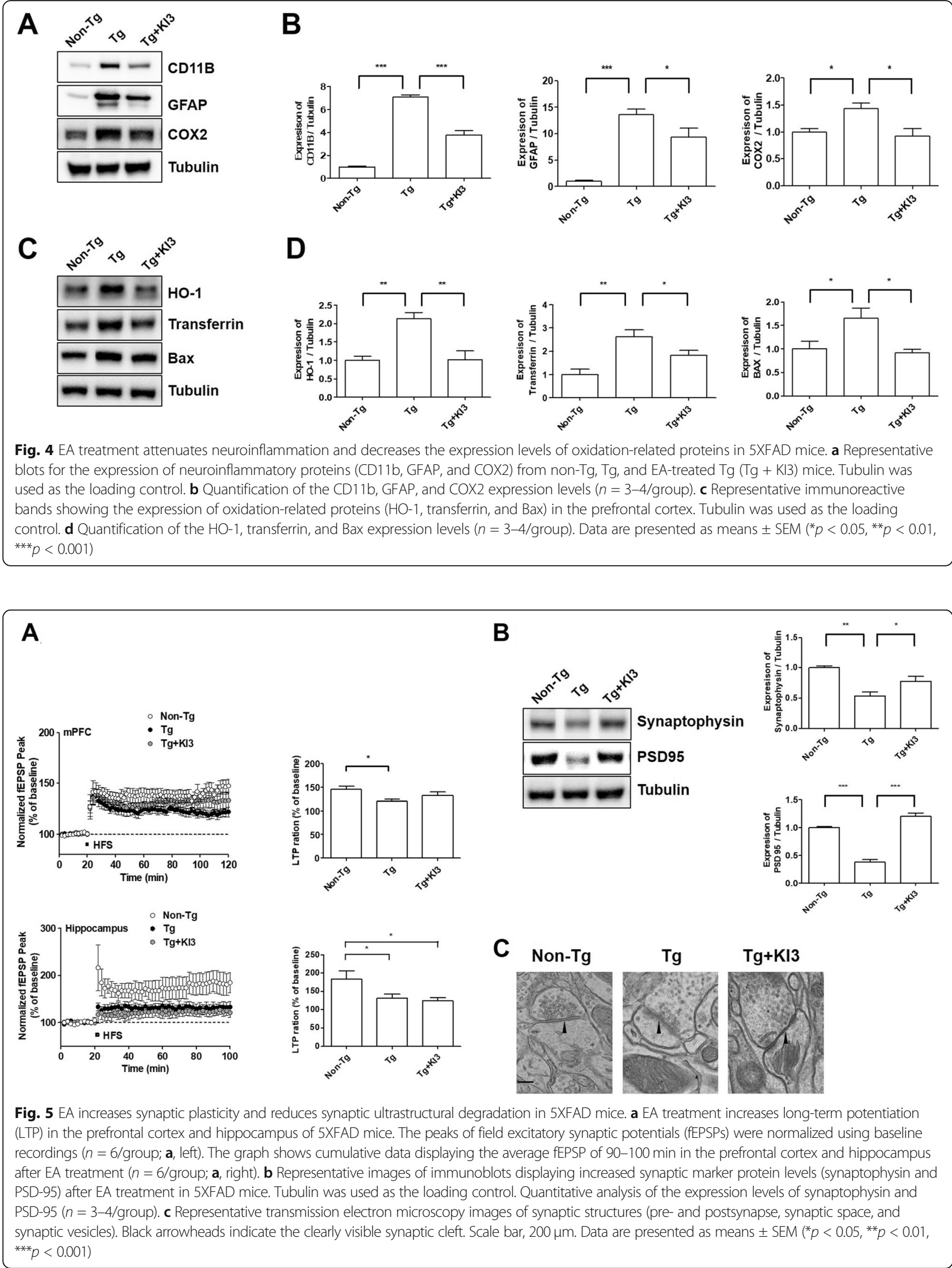


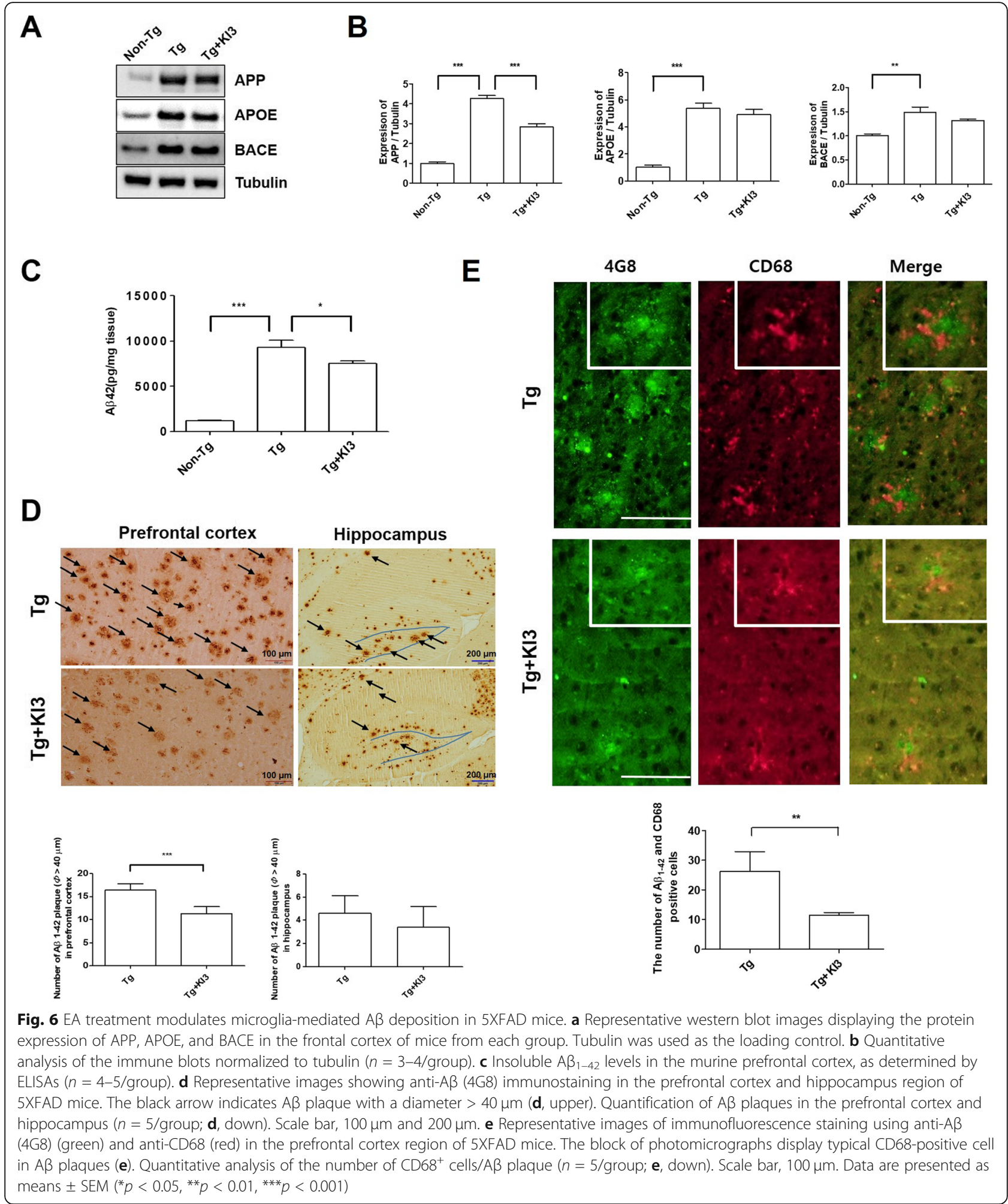

5XFAD mice to determine the relationship between a reduction in $A \beta$ plaque and neuroinflammation after $E A$ treatment. As shown in Fig. 6e, EA treatment significantly reduced 2.3-fold the number of colocalized $A \beta_{1-}$
42 and CD68 (microglia marker) in the prefrontal cortex of 5XFAD mice. These data indicate that EA treatment attenuates microglia-mediated $A \beta_{1-42}$ deposition in the prefrontal cortex of $\mathrm{Tg}$ mice. 


\section{Discussion}

In past decades, the incidence of cognitive impairment has been increasing due to various reasons, and many people focus now on the prevention of cognitive impairments that may lead to dementia. EA as one of the complementary alternative medicine (CAM) techniques showed clinically neuroprotective effects in patients with mild cognitive impairment [27]. In addition, the effectiveness of EA for cognitive improvement in $\mathrm{AD}$ and vascular dementia is supported by clinical and animal studies $[28,29]$. However, there has been no report yet on the mechanisms linking changes in cognition and neuroinflammation in EA-treated $\mathrm{AD}$ animals. In the current study, we revealed for the first time the EA-induced molecular mechanisms responsible for the correlation between cognitive improvement and anti-inflammation in the brains of 5XFAD mice, an animal model of AD.

5XFAD mice reveal hippocampus- or cortex-dependent cognitive impairments in behavioral tests such as the Ymaze test or contextual fear conditioning [30, 31]. The prefrontal cortex is important for recognition memory, facilitating the association between novel and familiar objects [32]. In our study, we confirmed that cognitive deficits in 5XFAD mice were hippocampus- and cortexdependent. In the NOR test, EA treatment significantly improved the hippocampus-independent working memory in 5XFAD mice. In hippocampus-dependent cognition, EA treatment showed a trend to effectiveness without reaching statistical significance. This indicates that a longer EA treatment may be necessary to reverse this impairment. These data suggest that EA treatment activates rather the prefrontal cortex area than the hippocampus in 5XFAD mice because only a short-term EA treatment for 2 weeks was performed in this study. In agreement with these data, Girard et al. reported that early cognitive deficits in 5XFAD mice are related to the frontal cortex before hippocampus-dependent impairments are detectable [31]. However, it needs to be investigated in further studies whether long-term EA treatment can also recover the hippocampus-dependent long-term memory.

Using microPET, we also observed that EA treatment activated the frontal cortex and the hypothalamus in 5XFAD mice. These results indicate that EA treatment modulates the abnormal hypothalamic metabolism and contributes to the energy metabolism, thus improving cognitive functions in 5XFAD mice. This is supported by Zheng et al. demonstrating that brain metabolism abnormalities are mainly detected in the hypothalamus and that these disturbances of the hypothalamus metabolism are related to the amyloid pathology in $\mathrm{AD}$ mice [33]. Recently, Wang et al. reported that adiponectin regulates glucose and lipid metabolism, as well as insulin sensitivity in the hypothalamus, hippocampus, and cerebral cortex, and improves cognitive functions by an increase in synaptic functions and LTP in 5XAFD mice [34]. In addition, they demonstrated that adiponectin treatment modulates apoptotic signaling including activated caspase 3 and nuclear factor kappa-light-chain-enhancer of activated $B$ cells (NF-kB) and glycogen synthase kinase 3 beta (GSK3 $\beta$ ) activation. Furthermore, Guo et al. showed that EA treatment increases the level of adiponectin production through adiponectin receptor 1-mediated GSK3 $\beta$ activation [35]. Therefore, we suggest that cortex and hypothalamus activation by EA treatment may increase adiponectin production and glucose metabolism thus leading to improved cognitive functions in 5XFAD mice.

Neuroinflammation, indicated by the presence of activated microglia, is a critical factor contributing to $\mathrm{AD}$ pathogenesis. Many markers including pro-inflammatory cytokines released by activated microglia result in degenerative changes of neurons and are associated with amyloid plaques [36]. Several papers demonstrate that EAinduced anti-inflammatory actions improve cognition and suppress neuroinflammatory factors such as TNF- $\alpha$ and IL-1 $\beta$ involved in toll-like receptor (TLR) signaling in animal models of vascular dementia $[19,37,38]$. In addition, Feng et al. show that EA treatment suppresses the expression levels of pro-inflammatory cytokines related to cognitive decline, including IL- $1 \alpha$, IL-6, and TNF- $\alpha$ [13]. Consistent with these publications, our data demonstrate that EA treatment attenuates neuroinflammation-related proteins (CD11b, GFAP, and COX), as well as oxidationrelated proteins (HO-1, transferrin, and $\mathrm{Bax}$ ), in the prefrontal cortex of 5XFAD mice. These data suggest that EA treatment can regulate synaptic plasticity and the synapse structure based on its modulation of neuroinflammation.

Synaptic plasticity regulates structures and functions of synapse [39]. In AD mice, a loss of synapses is observed and leads to learning and memory impairments [40]. Acupuncture improves spatial learning and memory and prevents changes in dendritic structures in the SAMP8 model [41]. Jing et al. show EA-induced improvement of cognition via LTP enhancement in a rat model of memory impairment [42]. In addition, Li et al. demonstrate that synaptic plasticity is regulated by $N$ methyl-D-aspartate receptor and mammalian target of rapamycin signaling pathways [43, 44]. In support of this, the current study reveals that EA treatment improves synaptic plasticity in 5XFAD mice via an increase in synaptic proteins such as synaptophysin and PSD-95, as well as via reduced ultrastructural degradation. Synaptophysin as a marker of presynaptic plasticity plays a critical role in the release of neurotransmitters, whereas PSD-95 is a postsynaptic marker [45]. Therefore, further studies are necessary to determine the signaling pathways involved in the neurotransmitter-dependent improvement of synaptic plasticity and cognition after EA treatment of 5XFAD mice.

In $\mathrm{AD}$ patients and the corresponding animal models, $\mathrm{A} \beta$ deposition is detected in both the hippocampus and the 
cerebral cortex, resulting in learning and memory impairments $[46,47]$. Neuroinflammation in $A D$ is related to $A \beta$ accumulation in glial cells such as microglia and astrocytes, thus affecting synaptic plasticity $[14,48]$. Activated microglia are involved in $A \beta$ production and clearance in the brain of $\mathrm{AD}$ mice [49]. Zhong et al. demonstrated in an animal model of $\mathrm{AD}$ that the microglial surface receptor triggering receptor expressed on myeloid cells 2 (TREM-2) plays as a critical role in the protection of neurons by enhancing microglial activities against $A \beta$ depositions and their related toxicity [50]. Based on our results, we conclude that EA treatment significantly decreases the amount of insoluble $A \beta$ in the prefrontal cortex of 5XFAD mice. Furthermore, we observed that EA treatment significantly reduced smaller and compact microglia than microglia colocalized with $\mathrm{A} \beta$ plaque in control 5XFAD even though the plaques in EA-treated 5XFAD mice are associated with the remaining microglia. Taken together, our data indicate that EA treatment reduces the number of microglia-mediated $A \beta$ plaques.

Vereker et al. showed that the pro-inflammatory cytokine IL-1 $\beta$ reduces LTP by inhibition of glutamate release in aged rats [51]. Moreover, Feng et al. demonstrated that EA treatment improves cognitive impairments by alleviating neuroinflammation via the microglial TLR2/4 signaling pathway in an animal model of post-operative cognitive dysfunction [51]. In the context of these studies, our data suggest that EA treatment promotes microglia-mediated $A \beta$ degradation and alleviates neuroinflammation via the reduction of proinflammatory cytokines in 5XFAD mice. In further studies, it remains to be shown whether the EA-induced alleviation of neuroinflammation and amyloid deposition is dependent on either the frequency of EA treatments or other parameters such as the duration of each session.

\section{Conclusions}

This study provides scientific evidence for anti-inflammatory effects in EA-treated 5XFAD mice, although it remains controversial whether anti-inflammation should be the main target to treat $\mathrm{AD}$ patients because anti-inflammatory drugs such as prednisone and rofecoxib failed in clinical studies with $\mathrm{AD}$ patients $[52,53]$. Reduction in neuroinflammation is the therapeutic target of $\mathrm{EA}$ in $\mathrm{AD}$, and EA may ameliorate $A \beta$-related pathologies and activate signaling pathways responsible for the improvement of cognitive functions in $\mathrm{AD}$ mice.

\section{Supplementary information}

Supplementary information accompanies this paper at https://doi.org/10. 1186/s12974-019-1665-3.

Additional file 1: Figure S1. Images of the full western blots from prefrontal cortex samples of 5XFAD mice. (a) HO-1, APP, Transferrin, and tubulin ( $n=3-4 /$ group). (b) CD11B, COX2, BAX, and tubulin ( $n=3-4$ / group). (c) BACE, APOE and tubulin ( $n=3-4 /$ group). (d) GFAP, PSD95 and tubulin ( $n=3-4$ /group). (e) Synaptophysin and tubulin ( $n=3-4 /$ group).)

\section{Abbreviations}

(HO)-1: Heme oxygenase; (TNF)-a: Tumor necrosis factor; 5XFAD: Five familial AD; AD: Alzheimer's disease; APOE: Apolipoprotein E; APP: Amyloid precursor protein; $A B$ : Amyloid beta; Bax: $B$ cell lymphoma-2 (BCl-2)-associated $X$ protein; CD1 1b: Cluster of differentiation 11 b; EA: Electroacupuncture; fEPSP: Field excitatory postsynaptic potential; GFAP: Glial fibrillary acidic protein; HFS: High-frequency stimulation; KI3: Taegye; LTP: Long-term potentiation; PSD-95: Postsynaptic density-95; ROI: Regions of interest; SUV: Standardized uptake value

\section{Acknowledgements}

We would like to thank Dr. A. Ram Ryu (KBIO HEALTH) for the help with the microPET imaging and its data analysis and Professor Dong Hyun Kim (Dong-A University), who helped with the electrophysiological experiments and their data analysis.

\section{Authors' contributions}

MDC performed all experiments and partially wrote the manuscript. JHL discussed the EA treatment and contributed to the data analysis. EJY designed this study, performed the TEM experiments, analyzed data, and provided the final manuscript. All authors read approved the final manuscript.

\section{Funding}

This study was supported by the Korea Institute of Oriental Medicine (KIOM) under Grant KSN1621051, and the Basic Science Research Program through the National Research Foundation of Korea funded by the Ministry of Science, ICT \& Future Planning, South Korea, under Grant NRF-2015R1C1A2A01053248.

\section{Availability of data and materials}

The datasets used and/or analyzed in the current study are available from the corresponding author on reasonable request.

\section{Ethics approval and consent to participate}

All experiments were approved by the IACUC of KIOM under the registration number 18-033.

\section{Consent for publication \\ Not applicable}

\section{Competing interests}

The authors declare that they have no competing interest.

\section{Author details}

'Department of Herbal Medicine Research, Korea Institute of Oriental Medicine, 1672 Yuseong-daero, Yuseong-gu, Daejeon 305-811, Republic of Korea. ${ }^{2}$ Department of Clinical Research, Korea Institute of Oriental Medicine, 1672 Yuseong-daero, Yuseong-gu, Daejeon 305-811, Republic of Korea.

Received: 28 June 2019 Accepted: 27 November 2019

Published online: 13 December 2019

\section{References}

1. Raskin J, Cummings J, Hardy J, Schuh K, Dean RA. Neurobiology of Alzheimer's disease: integrated molecular, physiological, anatomical, biomarker, and cognitive dimensions. Curr Alzheimer Res. 2015;12:712-22.

2. Bronzuoli MR, lacomino A, Steardo L, Scuderi C. Targeting neuroinflammation in Alzheimer's disease. J Inflamm Res. 2016:9:199-208.

3. Jimenez S, Baglietto-Vargas D, Caballero C, Moreno-Gonzalez I, Torres M, Sanchez-Varo R, Ruano D, Vizuete M, Gutierrez A, Vitorica J. Inflammatory response in the hippocampus of PS1M146L/APP751SL mouse model of Alzheimer's disease: age-dependent switch in the microglial phenotype from alternative to classic. J Neurosci. 2008;28:11650-61.

4. Krstic D, Madhusudan A, Doehner J, Vogel P, Notter T, Imhof C, Manalastas A, Hilfiker M, Pfister S, Schwerdel C, et al. Systemic immune challenges trigger and drive Alzheimer-like neuropathology in mice. J Neuroinflammation. 2012:9:151.

5. Hickman SE, Allison EK, El Khoury J. Microglial dysfunction and defective beta-amyloid clearance pathways in aging Alzheimer's disease mice. J Neurosci. 2008;28:8354-60 
6. Wyss-Coray T, Rogers J. Inflammation in Alzheimer disease-a brief review of the basic science and clinical literature. Cold Spring Harb Perspect Med. 2012;2:a006346.

7. Schafer DP, Stevens B. Microglia Function in Central Nervous System Development and Plasticity. Cold Spring Harb Perspect Biol. 2015;7:a020545.

8. Ullian EM, Sapperstein SK, Christopherson KS, Barres BA. Control of synapse number by glia. Science. 2001;291:657-61.

9. Falcao AS, Silva RF, Pancadas S, Fernandes A, Brito MA, Brites D. Apoptosis and impairment of neurite network by short exposure of immature rat cortical neurons to unconjugated bilirubin increase with cell differentiation and are additionally enhanced by an inflammatory stimulus. J Neurosci Res. 2007;85:1229-39.

10. Furman JL, Sama DM, Gant JC, Beckett TL, Murphy MP, Bachstetter AD, Van Eldik $L$, Norris CM. Targeting astrocytes ameliorates neurologic changes in a mouse model of Alzheimer's disease. J Neurosci. 2012:32:16129-40.

11. Li X, Guo F, Zhang Q, Huo T, Liu L, Wei H, Xiong L, Wang Q. Electroacupuncture decreases cognitive impairment and promotes neurogenesis in the APP/PS1 transgenic mice. BMC Complement Altern Med. 2014;14:37.

12. Lee B, Sur B, Shim J, Hahm DH, Lee H. Acupuncture stimulation improves scopolamine-induced cognitive impairment via activation of cholinergic system and regulation of BDNF and CREB expressions in rats. BMC Complement Altern Med. 2014;14:338.

13. Feng PP, Deng P, Liu LH, Ai Q, Yin J, Liu Z, Wang GM. Electroacupuncture alleviates postoperative cognitive dysfunction in aged rats by inhibiting hippocampal neuroinflammation activated via microglia/TLRs pathway. Evid Based Complement Alternat Med. 2017;2017:6421260.

14. Oakley H, Cole SL, Logan S, Maus E, Shao P, Craft J, Guillozet-Bongaarts A, Ohno M, Disterhoft J, Van Eldik L, et al. Intraneuronal beta-amyloid aggregates, neurodegeneration, and neuron loss in transgenic mice with five familial Alzheimer's disease mutations: potential factors in amyloid plaque formation. J Neurosci. 2006:26:10129-40.

15. Jeon SG, Cha MY, Kim Jl, Hwang TW, Kim KA, Kim TH, Song KC, Kim JJ, Moon M. Vitamin D-binding protein-loaded PLGA nanoparticles suppress Alzheimer's disease-related pathology in 5XFAD mice. Nanomedicine. 2019;17:297-307.

16. Lu KW, Yang J, Hsieh CL, Hsu YC, Lin YW. Electroacupuncture restores spatial learning and downregulates phosphorylated N-methyl-D-aspartate receptors in a mouse model of Parkinson's disease. Acupunct Med. 2017:35:133-41.

17. Cai M, Shin BY, Kim DH, Kim JM, Park SJ, Park CS, Won do H, Hong ND, Kang DH, Yutaka Y, Ryu JH. Neuroprotective effects of a traditional herbal prescription on transient cerebral global ischemia in gerbils. J Ethnopharmacol. 2011;138:723-30.

18. Bevins RA, Besheer J. Object recognition in rats and mice: a one-trial nonmatching-to-sample learning task to study 'recognition memory'. Nat Protoc. 2006;1:1306-11.

19. Yang EJ, Cai M, Lee JH. Neuroprotective effects of electroacupuncture on an animal model of bilateral common carotid artery occlusion. Mol Neurobiol. 2016;53:7228-36.

20. Cai M, Lee SH, Yang EJ. Bojungikgi-tang improves muscle and spinal cord function in an amyotrophic lateral sclerosis model. Mol Neurobiol. 2019;56: 2394-407.

21. Cai M, Lee $\mathbf{J H}$, Yang EJ. Bee venom ameliorates cognitive dysfunction caused by neuroinflammation in an animal model of vascular dementia. Mol Neurobiol. 2017;54:5952-60.

22. Chen $\mathrm{S}$, Xu M, Li H, Liang J, Yin L, Liu X, Jia X, Zhu F, Wang D, Shi X, Zhao L. Acupuncture at the Taixi (KI3) acupoint activates cerebral neurons in elderly patients with mild cognitive impairment. Neural Regen Res. 2014;9:1163-8.

23. Liu Z, Wei W, Bai L, Dai R, You Y, Chen S, Tian J. Exploring the patterns of acupuncture on mild cognitive impairment patients using regional homogeneity. PLoS One. 2014;9:e99335.

24. Minter MR, Taylor JM, Crack PJ. The contribution of neuroinflammation to amyloid toxicity in Alzheimer's disease. J Neurochem. 2016;136:457-74.

25. Jones RS, Lynch MA. How dependent is synaptic plasticity on microglial phenotype? Neuropharmacology. 2015;96:3-10.

26. Morgan D, Gordon MN, Tan J, Wilcock D, Rojiani AM. Dynamic complexity of the microglial activation response in transgenic models of amyloid deposition: implications for Alzheimer therapeutics. J Neuropathol Exp Neurol. 2005:64:743-53.

27. Tan $\Pi$, Wang D, Huang JK, Zhou XM, Yuan X, Liang JP, Yin L, Xie HL, Jia XY, Shi J, et al. Modulatory effects of acupuncture on brain networks in mild cognitive impairment patients. Neural Regen Res. 2017;12:250-8.
28. Leung MC, Yip KK, Ho YS, Siu FK, Li WC, Garner B. Mechanisms underlying the effect of acupuncture on cognitive improvement: a systematic review of animal studies. J Neuroimmune Pharmacol. 2014;9:492-507.

29. Zhao L, Zhang H, Zheng Z, Huang J. Electroacupuncture on the head points for improving gnosia in patients with vascular dementia. J Tradit Chin Med. 2009:29:29-34.

30. Hongpaisan J, Sun MK, Alkon DL. PKC epsilon activation prevents synaptic loss, Abeta elevation, and cognitive deficits in Alzheimer's disease transgenic mice. J Neurosci. 2011;31:630-43.

31. Girard SD, Baranger K, Gauthier C, Jacquet M, Bernard A, Escoffier G, Marchetti E, Khrestchatisky M, Rivera S, Roman FS. Evidence for early cognitive impairment related to frontal cortex in the 5XFAD mouse model of Alzheimer's disease. J Alzheimers Dis. 2013:33:781-96.

32. Zhou LYY, Wright TE, Clarkson AN. Prefrontal cortex stroke induces delayed impairment in spatial memory. Behav Brain Res. 2016;296:373-8.

33. Zheng H, Zhou Q, Du Y, Li C, Xu P, Lin L, Xiao J, Gao H. The hypothalamus as the primary brain region of metabolic abnormalities in APP/PS1 transgenic mouse model of Alzheimer's disease. Biochim Biophys Acta Mol Basis Dis. 2018;1864:263-73.

34. Wang M, Jo J, Song J. Adiponectin improves long-term potentiation in the 5XFAD mouse brain. Sci Rep. 2019:9:8918.

35. Guo F, Jiang T, Song W, Wei H, Wang F, Liu L, Ma L, Yin H, Wang Q, Xiong L. Electroacupuncture attenuates cerebral ischemia-reperfusion injury in diabetic mice through adiponectin receptor 1-mediated phosphorylation of GSK-3beta. Mol Neurobiol. 2015;51:685-95.

36. Eikelenboom P, Veerhuis R. The role of complement and activated microglia in the pathogenesis of Alzheimer's disease. Neurobiol Aging. 1996:17:673-80.

37. Sugaya K, Chouinard M, Greene R, Robbins M, Personett D, Kent C, Gallagher M, McKinney M. Molecular indices of neuronal and glial plasticity in the hippocampal formation in a rodent model of age-induced spatial learning impairment. J Neurosci. 1996;16:3427-43.

38. Li W, Lai XS. Changes of interleukin-1 beta and TNF-alpha contents in the hippocampus and the interventional effect of electroacupuncture in vascular dementia rats. Zhen Ci Yan Jiu. 2007;32:34-7.

39. Lohmann C, Kessels HW. The developmental stages of synaptic plasticity. J Physiol. 2014;592:13-31.

40. Forner S, Baglietto-Vargas D, Martini AC, Trujillo-Estrada L, LaFerla FM. Synaptic impairment in Alzheimer's disease: a dysregulated symphony. Trends Neurosci. 2017:40:347-57.

41. Kan BH, Yu JC, Zhao L, Zhao J, Li Z, Suo YR, Han JX. Acupuncture improves dendritic structure and spatial learning and memory ability of Alzheimer's disease mice. Neural Regen Res. 2018;13:1390-5.

42. Jing $X H$, Chen SL, Shi H, Cai H, Jin ZG. Electroacupuncture restores learning and memory impairment induced by both diabetes mellitus and cerebral ischemia in rats. Neurosci Lett. 2008;443:193-8.

43. Li F, Tsien JZ. Memory and the NMDA receptors. N Engl J Med. 2009;361: 302-3.

44. Swiech L, Perycz M, Malik A, Jaworski J. Role of mTOR in physiology and pathology of the nervous system. Biochim Biophys Acta. 2008;1784:116-32.

45. Greengard P, Valtorta F, Czernik AJ, Benfenati F. Synaptic vesicle phosphoproteins and regulation of synaptic function. Science. 1993;259: 780-5.

46. McGowan E, Eriksen J, Hutton M. A decade of modeling Alzheimer's disease in transgenic mice. Trends Genet. 2006:22:281-9.

47. Gotz J, Gotz NN. Animal models for Alzheimer's disease and frontotemporal dementia: a perspective. ASN Neuro. 2009;1.

48. Ben Achour S, Pascual O. Glia: the many ways to modulate synaptic plasticity. Neurochem Int. 2010;57:440-5.

49. Baik SH, Kang S, Son SM, Mook-Jung I. Microglia contributes to plaque growth by cell death due to uptake of amyloid beta in the brain of Alzheimer's disease mouse model. Glia. 2016:64:2274-90.

50. Zhong L, Xu Y, Zhuo R, Wang T, Wang K, Huang R, Wang D, Gao Y, Zhu Y, Sheng $X$, et al. Soluble TREM2 ameliorates pathological phenotypes by modulating microglial functions in an Alzheimer's disease model. Nat Commun. 2019:10:1365

51. Vereker E, O'Donnell E, Lynch MA. The inhibitory effect of interleukin-1beta on long-term potentiation is coupled with increased activity of stressactivated protein kinases. J Neurosci. 2000;20:6811-9.

52. Koch HJ, Szecsey A. A randomized controlled trial of prednisone in Alzheimer's disease. Neurology. 2000;55:1067. 
53. Thal $L$, Ferris $S H$, Kirby L, Block GA, Lines CR, Yuen E, Assaid C, Nessly ML, Norman BA, Baranak CC, et al. A randomized, double-blind, study of rofecoxib in patients with mild cognitive impairment. Neuropsychopharmacology. 2005;30:1204-15.

\section{Publisher's Note}

Springer Nature remains neutral with regard to jurisdictional claims in published maps and institutional affiliations.

Ready to submit your research? Choose BMC and benefit from:

- fast, convenient online submission

- thorough peer review by experienced researchers in your field

- rapid publication on acceptance

- support for research data, including large and complex data types

- gold Open Access which fosters wider collaboration and increased citations

- maximum visibility for your research: over $100 \mathrm{M}$ website views per year

At $B M C$, research is always in progress. 